Service social

\title{
Taux de récurrence chez les enfants signalés à une direction de la protection de la jeunesse entre 1998 et 2002
}

\author{
Danny Dessureault, Louise S. Éthier, Tristan Milot et Martin Dionne
}

Volume 51, numéro 1, 2004

URI : https://id.erudit.org/iderudit/012708ar

DOI : https://doi.org/10.7202/012708ar

Aller au sommaire du numéro

Éditeur(s)

École de service social de l'Université Laval

ISSN

1708-1734 (numérique)

Découvrir la revue

Citer cet article

Dessureault, D., Éthier, L. S., Milot, T. \& Dionne, M. (2004). Taux de récurrence chez les enfants signalés à une direction de la protection de la jeunesse entre 1998 et 2002. Service social, 51(1), 1-13. https://doi.org/10.7202/012708ar
Résumé de l'article

L'application de la Loi sur la protection de la jeunesse repose sur le signalement d'une situation qui compromet la sécurité ou le développement d'un enfant. Depuis quelques années les intervenants des centres jeunesse constatent qu'un nombre élevé d'enfants font l'objet d'un nouveau signalement au fil des ans : on parle alors de récurrence. Les études menées sur ce sujet rapportent des taux de récurrence variant entre $18 \%$ et $60 \%$. Or, une majorité d'études s'appuient sur de petits échantillons, et non sur des cohortes, pour établir les taux de récurrence. L’objectif de la présente étude est d'estimer la récurrence en s'appuyant sur cinq cohortes d'enfants $(n=8738)$ dont le signalement a été analysé par la direction de la protection de la jeunesse d'un centre jeunesse entre 1998 et 2002. Les résultats indiquent que $24,5 \%$ des enfants évalués avaient fait l'objet d'une enquête antérieurement et qu'un enfant sur six (15,8 \%) avait reçu des services des centres jeunesse. Finalement, les prévalences les plus fortes s'observent chez les garçons, dans le groupe d'âge des 13-17 ans et chez ceux qui sont signalés pour une problématique d'abandon et de négligence. 


\title{
Taux de récurrence chez les enfants signalés à une direction de la protection de la jeunesse entre 1998 et $2002^{1}$
}

\author{
Danny DESSUREAULT \\ Professeur \\ Département de psychoéducation \\ Université du Québec à Trois-Rivières \\ Louise S. ÉTHIER \\ Professeure \\ Département de psychologie \\ Université du Québec à Trois-Rivières \\ Tristan MILOT \\ Étudiant au doctorat \\ Département de psychologie \\ Université du Québec à Trois-Rivières \\ Martin DIONNE \\ Centres jeunesse de la Mauricie et du Centre-du-Québec
}

L'application de la Loi sur la protection de la jeunesse repose sur le signalement d'une situation qui compromet la sécurité ou le développement d'un enfant. Depuis quelques années les intervenants des centres jeunesse constatent qu'un nombre élevé d'enfants font l'objet d'un nouveau signalement au fil des ans : on parle alors de récurrence. Les études menées sur ce sujet rapportent des taux de récurrence variant entre $18 \%$ et $60 \%$. Or, une majorité d'études s'appuient sur de petits échantillons, et non sur des cohortes, pour établir les taux de récurrence. L'objectif de la présente étude est d'estimer la récurrence en s'appuyant sur cinq cohortes d'enfants $(n=8738)$ dont le signalement a été analysé par la direction de la protection de la jeunesse d'un centre jeunesse entre 1998 et 2002. Les résultats indiquent que $24,5 \%$ des enfants évalués avaient fait l'objet d'une enquête antérieurement et qu'un enfant sur six (15,8\%) avait

1. Cette recherche a été rendue possible grâce à Valorisation-Recherche Québec, dans le cadre de l'élaboration de la "Plate-forme informationnelle pour le bien-être de l'enfant », et grâce au Fonds institutionnel de recherche de l'Université du Québec à Trois-Rivières. Les auteurs tiennent à remercier le Centre jeunesse de la Mauricie et du Centre-du-Québec de leur avoir donné accès à leurs données et en particulier MM. Yves Mercure et Daniel Gagnon pour leur précieuse collaboration.

Service social, volume 51, numéro 1, 2004-2005 
reçu des services des centres jeunesse. Finalement, les prévalences les plus fortes s'observent chez les garçons, dans le groupe d'âge des 13-17 ans et chez ceux qui sont signalés pour une problématique d'abandon et de négligence.

Mots clés : enfants, récurrence, cohorte, direction de la protection de la jeunesse.

Over the past few years, caseworkers in Child Protection Centres in Quebec have noticed a recurrence of referrals for a significant number of children over time. Recent estimates of recurrence for children who received child protection services (CPS) range from $18 \%$ to $60 \%$. Most studies base their rate on small samples or cases referred over a short period of time, and not on cohorts of CPS-evaluated children. The focus of this study is to estimate the recurrence rate using data from 5 cohorts of children referred and evaluated $(n=8738)$ by a CPS between 1998 and 2002. Results show that $24.5 \%$ of substantiated cases had been evaluated in the past by CPS, and one out of six children (15.8\%) had received CPS services. Boys, children in the 1317 age group and cases referred for neglect or abandonment showed the highest recurrence rate.

Key words: children, recurrence, cohort, child protection services.

\section{INTRODUCTION}

Le gouvernement du Québec adoptait en 1977 la Loi sur la protection de la jeunesse (LPJ). Essentiellement, cette loi a pour but d'assurer la protection des enfants (0 à 18 ans) dont la sécurité ou le développement peut être compromis, c'est-à-dire des enfants qui vivent des situations intolérables engendrant des conséquences majeures sur leur sécurité ou leur développement global. Le processus d'application de la loi repose sur le signalement d'une situation de compromission à laquelle une personne a un motif raisonnable de croire qu'un enfant est exposé. Trois étapes sont prévues pour le traitement des signalements. Dès la divulgation de la situation à une direction de la protection de la jeunesse (DPJ), la première étape (réception) consiste à décider si le signalement doit être retenu pour une enquête plus approfondie au regard des allégations portées. À la seconde étape (évaluation), le directeur de la protection de la jeunesse (DPJ) doit statuer, après enquête, si la sécurité ou le développement de l'enfant est compromis. Dans la mesure où la compromission est établie, la dernière étape (orientation) consiste à offrir à l'enfant et à sa famille des mesures d'aide afin que la situation ne se reproduise plus ou ne menace plus la sécurité et le développement de l'enfant. Selon les données du ministère de la Santé et des Services sociaux, moins du quart de tous les signalements reçus sont retenus à l'étape « orientation » pour cause de compromission de la sécurité ou du développement d'un enfant (Lessard, 2001). Malgré les procédures et l'aide fournie par les centres jeunesse, il arrive que des enfants soient signalés à nouveau à la Direction de la protection de la jeunesse, la récurrence étant le terme employé pour rendre compte de ce phénomène.

Depuis quelques années, les intervenants à la DPJ constatent que de plus en plus d'enfants sont signalés ou, pire encore, « re-signalés » à leur service. La perception 
des intervenants à l'égard de l'évolution des signalements reçus et traités est corroborée depuis un certain temps par les données colligées par le ministère de la Santé et des Services sociaux. Ainsi, entre 1996 et 2001, la proportion des signalements traités par 1000 enfants sur l'ensemble du territoire québécois est passée de 28,4 à 33,8, soit une augmentation de $19 \%$ en cinq ans (Lessard, 2002). Une tendance similaire est aussi observée dans les services de protection de l'enfance en Ontario. Le taux des enquêtes sur les mauvais traitements envers les enfants, qui était de 20,5 par 1000 enfants en 1993, se situe à 27,5 en 1998 (Trocmé, Fallon, MacLaurin et Copp, 2002). Ce taux est inférieur aux taux enregistrés au Québec (Lessard, 2002). Toutefois, l'étude de Trocmé et al. (2002) ne considère pas les jeunes qui sont signalés pour des troubles de comportement, alors que les données québécoises englobent toutes les situations de compromission mentionnées par le législateur (articles 38 et 38.1 de la LPJ), y compris par le fait même les troubles de comportement.

En ce qui a trait à la récurrence observée à la protection de la jeunesse, très peu de travaux de recherche au Québec ont abordé ce phénomène depuis l'adoption de la loi. En 1994, Sheriff et Berlinguet ont réalisé la première étude québécoise sur la récurrence des signalements à la DPJ. En s'appuyant sur un échantillon de 295 dossiers retenus pour évaluation en 1993-1994 par la DPJ du Centre de services sociaux de Québec, elles ont observé que $18 \%$ des dossiers actifs en protection de la jeunesse et $24,6 \%$ des dossiers fermés se retrouvent en récurrence. La sommation de ces deux taux leur permet d'établir à 42,6\% le taux annuel de récurrence. Ainsi, $18 \%$ des enfants qui ont reçu des services durant l'année 1993-1994 ont fait l'objet d'un nouveau signalement au cours de la même année, alors que $24,6 \%$ de tous ceux qui avaient reçu des services antérieurement seront signalés à nouveau durant cette période. Toutefois, nous considérons que cette sommation des deux taux est plus ou moins pertinente, car l'addition des prévalences est basée à la fois sur des données rétrospectives et sur des données prospectives. Par ailleurs, les auteures de l'étude notent que la récurrence varie selon le groupe d'âge, c'est-à-dire qu'elle touche plus les adolescents de 12 à 17 ans (51\%) que les enfants âgés de 0 à 5 ans (26\%) ou de 6 à 11 ans (23\%). Plus récemment, l'étude de Jacob et Laberge (2001) soulignait que, parmi les signalements évalués en 1994-1995 par le Centre jeunesse de Québec $(n=270)$, $26,3 \%$ concernaient des dossiers inactifs, donc en situation de récurrence.

Bien que l'organisation des services de protection de l'enfance soit quelque peu différente ailleurs au Canada, l'étude canadienne sur l'incidence des signalements de cas de violence et de négligence (Trocmé, MacLaurin, Fallon, Daciuk, Billingsley, Tourigny et al., 2001) établit que $51 \%$ des enfants qui ont fait l'objet d'une enquête en 1998 pour des situations de maltraitance se retrouvent en situation de récurrence. Du côté des États-Unis, des estimations récentes indiquent que les taux de récurrence pour la maltraitance varient entre $18 \%$ et $60 \%$ (Inkelas et Halfon, 1997; English, Marshall, Brummel et Orme, 1999), bien qu'une majorité d'études situent ce taux autour de $50 \%$ (DePanfilis et Zuravin, 1999). Un taux similaire de récurrence de $40 \%$ est aussi observé au Royaume-Uni (Hamilton et Browne, 1999). 
Au regard des études citées, des remarques peuvent être formulées tant sous l'angle de la pratique sociale que de celui de la méthode. D'un point de vue de la pratique sociale, il faut souligner que les données qui permettent d'établir les taux de récurrence sont naturellement issues de processus de mise en application des lois visant la protection de l'enfance, et donc forcément tributaires du contexte social dans lequel s'inscrit ce processus. Or, le contexte social est par nature dynamique. II évolue selon les valeurs prédominantes à une période donnée, les choix politiques ou sociétaux ou, encore, la disponibilité des ressources humaines et financières pour assurer l'offre de service; autant d'éléments contextuels qui influencent directement ou indirectement les critères de rétention et d'évaluation des signalements par les agences de protection à l'enfance. À cet égard, Fortin, Lafleur et Robitaille (2002) constatent, sur le plan de l'organisation des services, que la majeure partie des ressources sont mobilisées par les services spécialisés à l'enfance, qui sont d'ailleurs régulièrement engorgés, bien qu'ils s'adressent à une faible proportion de jeunes. Qui plus est, Smith et Donovan (2003) ont observé au cours des dix dernières années que les changements administratifs et les politiques sociales engendrent un climat organisationnel axé essentiellement sur la transformation et la rentabilité des services sociaux, contexte qui favorise la recherche à tout prix de la pratique efficace en protection à l'enfance tout en s'inscrivant à l'intérieur d'une réduction du temps d'intervention. Ces pressions organisationnelles font que les intervenants portent une moins grande attention au travail avec les parents, se centrent davantage sur les familles qui sont disposées à recevoir de l'aide, tout en attribuant l'échec à la résistance parentale (Brodkin, 1997). La compréhension des taux de récurrence doit nécessairement tenir compte, en filigrane, de ces éléments contextuels.

Sous l'angle de la méthode, il est difficile de comparer les taux de récurrence dans les services de protection à l'enfance à cause des disparités sur les définitions employées pour opérationnaliser et mesurer la récurrence. Premièrement, certains auteurs considèrent la récurrence comme étant simplement le re-signalement d'un enfant (récurrence individuelle) ou d'un membre de la fratrie de cet enfant (récurrence familiale), alors que d'autres établissent leur taux en fonction d'une des étapes du processus d'évaluation des signalements par les services de protection à l'enfance (rétention, évaluation, compromission établie ou pas). À cette multiplicité des définitions s'ajoute la durée d'observation sur laquelle portent les études, laquelle peut aller de quelques semaines à plusieurs années. II s'avère donc difficile d'établir une comparaison équitable entre les divers résultats publiés à ce jour. Deuxièmement, les taux de récurrence obtenus par les études s'appuient généralement sur de petits échantillons ou sur des cohortes ne couvrant qu'une période restreinte de temps. Ces stratégies méthodologiques entraînent indéniablement une erreur d'échantillonnage sur l'estimation du taux de récurrence (Morin, 1993). Troisièmement, certaines recherches ne ciblent que les cas de maltraitance pour évaluer la récurrence, alors que d'autres englobent plusieurs situations de compromission (abandon, négligence, abus et troubles de comportement). Finalement, très peu d'études ventilent les taux de récurrence selon les caractéristiques des enfants ou les motifs de re-signalement. 


\section{OBJECTIFS}

Puisque notre étude est essentiellement descriptive, son objectif est d'abord de déterminer le taux de récurrence pour les cinq années comprises entre 1998 et 2002, puis de le ventiler en fonction du sexe et de l'âge de l'enfant ainsi que des situations retenues lors de l'évaluation par le directeur de la protection de la jeunesse. La réunion de ces différents taux de récurrence offrira pour une des premières fois une vue d'ensemble homogène sur une période d'observation de cinq ans.

\section{MÉTHODE}

\section{PARTICIPANTS}

Cinq cohortes sont constituées sur la base de chacune des cinq années comprises dans l'étude. Chaque cohorte regroupe tous les enfants âgés de moins de 18 ans dont le signalement est retenu par la DPJ des Centres jeunesse de la Mauricie et du Centre-du-Québec (CJMCQ) pour évaluation durant l'année visée, quel que soit le motif du signalement (négligence, abus, troubles de comportement, etc.). Les CJMCQ couvrent une vaste région géographique à l'intérieur de laquelle on retrouve des populations urbaines et rurales. Dans les cas où un enfant serait signalé plus d'une fois durant la même année, seul le dernier signalement évalué est considéré. Une telle procédure permet de vérifier, lors de l'application des critères d'inclusion pour déterminer une situation de récurrence, si le dossier de l'enfant est à ce moment soit fermé, soit actif. Entre 1998 et 2002, la DPJ du centre jeunesse a évalué 8738 enfants qui se répartissent comme suit dans chaque cohorte : 1610 enfants évalués en 1998, 1622 en 1999, 1598 en 2000, 1846 en 2001 et 2062 en 2002.

\section{SOURCE D'INFORMATION}

La réalisation de la présente étude s'appuie sur la base de données PIBE (Plate-forme informationnelle pour le bien-être de l'enfant) des CJMCQ. PIBE contient un ensemble standardisé d'informations administratives et cliniques dénominalisées à l'égard des signalements retenus et évalués par la DPJ d'un centre jeunesse. Techniquement, PIBE est une réplique partielle du Système Clientèle Jeunesse, système informatique implanté dans les centres jeunesse du Québec. Le système clientèle représente en quelque sorte le dossier électronique de l'usager, dont l'accès n'est autorisé qu'aux intervenants qui sont parties au dossier et dans lequel toute information nouvelle et pertinente, concernant l'usager et les services qu'il reçoit, est versée par ces derniers.

\section{DÉFINITION DE LA RÉCURRENCE}

Pour qu'un enfant soit considéré comme étant en situation de récurrence, l'étude impose certains critères. Ainsi, pour une année donnée, il faut qu'au moins un signalement concernant l'enfant ait fait l'objet d'une enquête au cours de la seconde étape du processus d'évaluation par la DPJ et que le dossier relatif à ce signalement soit fermé au moment de l'enquête sur un nouveau signalement durant l'année considérée. 


\section{RÉSULTAT}

Entre 1998 et 2002, le nombre d'enfants évalués pour un signalement retenu par la DPJ est passé de 1610 à 2 062, soit une augmentation de $28 \%$ en cinq ans, ce qui est bien au-delà de l'augmentation nationale observée (Lessard, 2002). Cette hausse est encore plus surprenante lorsque l'on considère l'ampleur des restrictions budgétaires imposées aux établissements du réseau de la santé et des services sociaux durant ces années.

\section{LES SIGNALEMENTS ÉVALUÉS PAR LA DPJ}

Les résultats obtenus et présentés au tableau 1 montrent que les évaluations effectuées entre 1998 et 2002 concernent significativement plus souvent les garçons $(52,4 \%)$ que les filles $(47,6 \%)\left(\chi^{2}(1, n=8738)=19,80, p<0,05\right)$. Une telle différence significative est aussi observée pour les cohortes de 1998 et de 2001 . Par ailleurs, les trois quarts des évaluations effectuées par la DPJ portent sur des enfants des groupes d'âge 6 à 12 ans et 13 à 17 ans, proportion qui demeure constante à l'intérieur des cinq cohortes. L'abandon et la négligence envers les enfants restent la situation la plus régulièrement évaluée par la $D P J$, puisqu'elles représentent près de la moitié de toutes les évaluations effectuées au cours des années. Plus précisément, 75,8\% de tous les enfants dont le motif d'évaluation est une situation d'abandon ou de négligence appartiennent aux groupes d'âge 0 à 5 ans (27,3\%) et 6 à 12 ans (48,5\%), alors que les troubles de comportement s'observent à 89,2\% chez les 13 à 17 ans. En ce qui regarde les situations d'exploitation ou d'abus, la prévalence moyenne la plus élevée $(55,2 \%)$ se situe chez les 6 à 12 ans, mais elle est tout de même de 14,9\% chez les 0 à 5 ans et de 29,9\% pour le groupe des 13 à 17 ans. Enfin, la comparaison, entre les prévalences des caractéristiques de la clientèle évaluée par la DPJ des CJMCQ et les données provinciales sur l'application de la LPJ (Lessard, 2002), montre une très forte similitude au regard du sexe, du groupe d'âge et de la situation retenue pour évaluation. Bien que ce ne soit pas un objectif implicitement poursuivi par cette étude, une généralisation de nos résultats à l'ensemble de la clientèle des DPJ du Québec pourrait être considérée, mais avec prudence.

En ce qui concerne la décision du DPJ, le tableau 1 indique, et cela demeure constant entre 1998 et 2002, que les évaluateurs, à la suite de leur enquête, établissent que la sécurité ou le développement est compromis (SDC) pour plus d'un enfant sur deux $(51,6 \%)$. Au regard de la période d'observation entre 1998 et 2002, il s'agit d'une augmentation de $23 \%$ du nombre d'évaluations se concluant en SDC. En contrepartie, les décisions statuant que la sécurité ou le développement est non compromis (SDNC) ont augmenté de $34 \%$ durant cette même période. Enfin, le taux moyen d'enfants qui se retrouvent en situation de récurrence est de 19,6\%. C'est donc un enfant sur cinq qui, au moment de la présente enquête, avait été antérieurement évalué par la DPJ. Qui plus est, dans la mesure où l'on ne tient compte que des évaluations antérieures concluant à la compromission et entraînant par le fait même une intervention psychosociale auprès de l'enfant et de sa famille, le taux moyen de récurrence est de 12,9\%, soit un enfant sur huit. 
Tableau 1

Caractéristiques des enfants, taux de récurrence et décision sur la compromission des signalements évalués par la Direction de la protection de la jeunesse (DPJ) au regard des cinq cohortes

\begin{tabular}{|c|c|c|c|c|c|c|c|c|c|c|c|}
\hline & \multicolumn{2}{|c|}{1998} & \multicolumn{2}{|c|}{1999} & \multicolumn{2}{|c|}{2000} & \multicolumn{2}{|c|}{2001} & \multicolumn{2}{|c|}{2002} & \multirow{2}{*}{$\begin{array}{c}\begin{array}{c}\text { Taux } \\
\text { moyen }\end{array} \\
\% \\
\end{array}$} \\
\hline & $\mathrm{n}$ & $\%$ & $\mathrm{n}$ & $\%$ & $\mathrm{n}$ & $\%$ & $\mathrm{n}$ & $\%$ & $\mathrm{n}$ & $\%$ & \\
\hline Caractéristiques des enfants évalués & 1610 & & 1622 & & 1598 & & 1846 & & 2062 & & \\
\hline Sexe & & & & & & & & & & & \\
\hline masculin & 849 & $52,7^{*}$ & 837 & 51,6 & 836 & 52,3 & 984 & $53,3^{* *}$ & 1071 & 51,9 & $52,4^{\star \star}$ \\
\hline féminin & 761 & 47,3 & 785 & 48,4 & 762 & 47,7 & 862 & 46,7 & 991 & 48,1 & 47,6 \\
\hline \multicolumn{12}{|l|}{ Groupe d'âge } \\
\hline 0 à 5 ans & 442 & 27,5 & 389 & 24,0 & 404 & 25,3 & 430 & 23,3 & 534 & 25,9 & 25,2 \\
\hline 6 à 12 ans & 567 & 35,2 & 623 & 38,4 & 572 & 35,8 & 659 & 35,7 & 752 & 36,5 & 36,3 \\
\hline 13 à 17 ans & 601 & 37,3 & 610 & 37,6 & 622 & 38,9 & 757 & 41,0 & 776 & 37,6 & 38,5 \\
\hline \multicolumn{12}{|l|}{ Situation retenue pour évaluation (alinéa 38) } \\
\hline abandon et négligence & 856 & 53,2 & 813 & 50,1 & 776 & 48,6 & 864 & 46,8 & 1069 & 51,8 & 50,1 \\
\hline exploitation et abus physique / sexuel & 302 & 18,8 & 338 & 20,8 & 365 & 22,8 & 380 & 20,6 & 456 & 22,1 & 21,1 \\
\hline trouble de comportement & 452 & 28,0 & 471 & 29,1 & 457 & 28,6 & 602 & 32,6 & 537 & 26,1 & 28,8 \\
\hline En situation de récurrence & 309 & 19,2 & 311 & 19,2 & 328 & 20,5 & 362 & 19,6 & 406 & 19,7 & 19,6 \\
\hline Récurrence avec évaluation antérieure SDC & 208 & 12,9 & 207 & 12,8 & 211 & 13,2 & 234 & 12,8 & 267 & 13,0 & 12,9 \\
\hline \multicolumn{12}{|l|}{ Conclusion de l'évaluation : } \\
\hline Sécurité / développement non compromis & 783 & 48,6 & 863 & 53,2 & 815 & 51,0 & 1001 & 54,2 & 1049 & 50,9 & 51,6 \\
\hline Sécurité / développement compromis & 827 & 51,4 & 759 & 46,8 & 783 & 49,0 & 845 & 45,8 & 1013 & 49,1 & 48,4 \\
\hline
\end{tabular}




\section{LES ENFANTS DONT LA SÉCURITÉ OU LE DÉVELOPPEMENT N'EST PAS COMPROMIS (SDNC)}

La première section du tableau 2 présente les caractéristiques de ces enfants qui se retrouvent en situation de récurrence. Ainsi, 15,1\% des enfants dont la sécurité ou le développement ne sont pas jugés compromis avaient déjà été évalués par la DPJ, les taux variant entre $12,3 \%$ et 16,9\% à l'intérieur des cinq cohortes. Qui plus est, pour plus des deux tiers de ces enfants, les enquêtes antérieures avaient statué qu'ils se trouvaient à ce moment dans une situation de compromission. Pour le centre jeunesse, il s'agit bien d'enfants connus et auprès desquels il était déjà intervenu, mais dont la situation actuelle n'est pas jugée compromettante sur le plan de la sécurité ou du développement. Parmi les enfants en situation de récurrence dont l'évaluation est SDNC (sécurité ou développement non compromis), les garçons sont présents dans une proportion de 53,6 \%, alors que la proportion des filles est de 46,4\%. Un tel écart n'est toutefois pas statistiquement significatif. Par ailleurs, $81,4 \%$ des enfants avec une évaluation SDNC et en récurrence appartiennent aux groupes d'âge des 6-12 ans et 13-17 ans et, dans 50,8\% des cas, l'évaluation jugée SDNC portait sur une problématique d'abandon ou de négligence parentale. 
Tableau 2

Taux de récurrence et caractéristiques des enfants selon que l'évaluation du signalement conclut que la sécurité ou le développement est non compromis (SDNC) ou compromis (SDC)

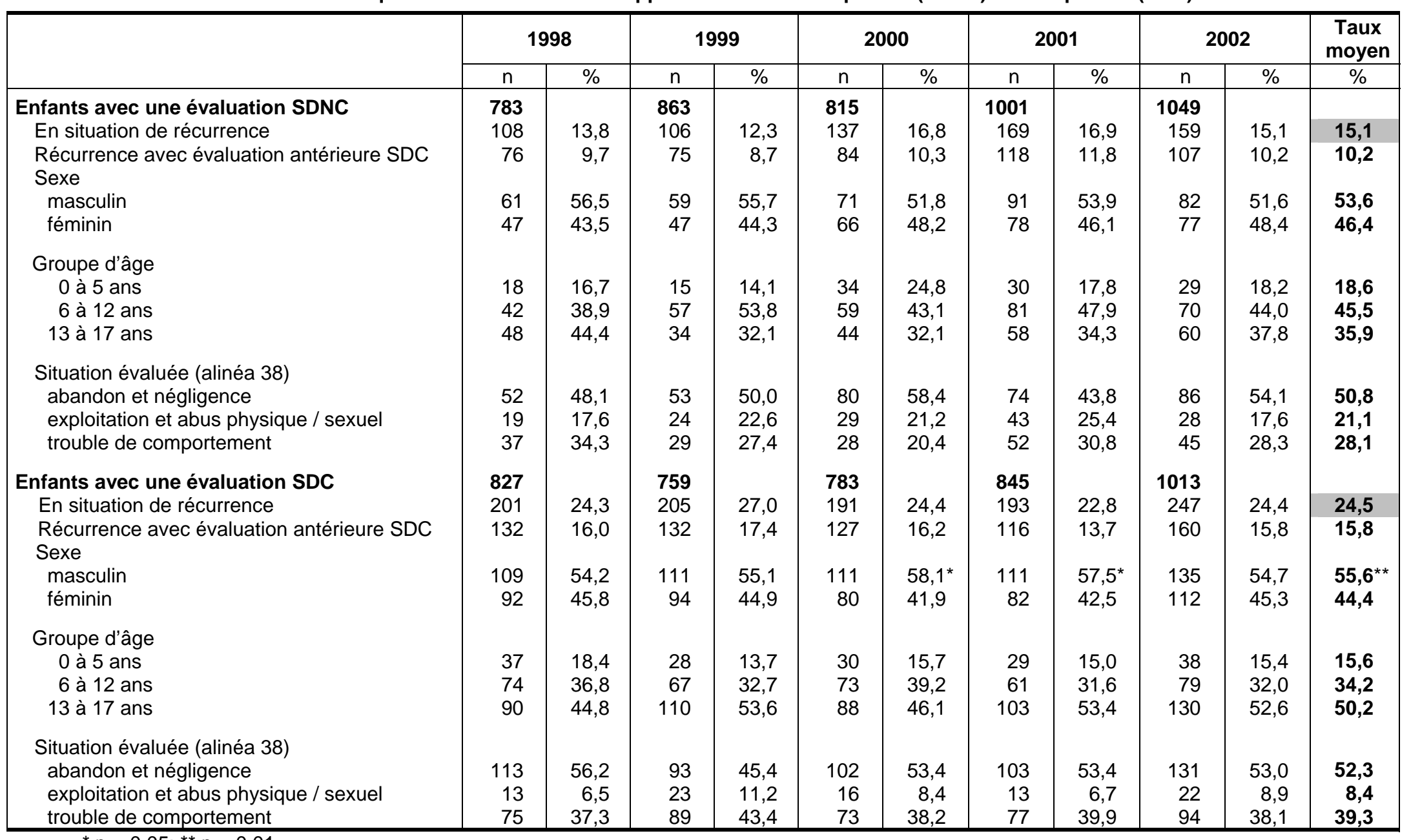




\section{LES ENFANTS DONT LA SÉCURITÉ OU LE DÉVELOPPEMENT EST COMPROMIS (SDC)}

Comme on peut le voir au tableau 2, près d'un enfant sur quatre $(24,5 \%)$ se retrouve en situation de récurrence à la suite d'une évaluation concluant que sa sécurité ou son développement est compromis (SDC). Et si l'on tient compte du fait qu'au moins une évaluation antérieure avait conclu à la sécurité ou au développement compromis, c'est un enfant sur six $(15,8 \%)$ auprès duquel le centre jeunesse était intervenu qui se retrouve en récurrence. Sur le plan des caractéristiques, on constate que les garçons sont significativement plus nombreux $(55,6 \%)$ que les filles $(44,4 \%)$ à se retrouver en récurrence. D'ailleurs, cette différence significative s'observe aussi à l'intérieur des cohortes de 2000 et de 2001, bien qu'une tendance similaire, mais non significative, se manifeste dans les cohortes de 1998, de 1999 et de 2002. Au regard du groupe d'âge, $84,4 \%$ des enfants en récurrence font partie des 6-12 ans (34,2\%) ou des 13-17 ans (50,2 \%). Par comparaison avec les enfants dont la sécurité ou le développement n'est pas compromis, les proportions pour les groupes d'âge sont inversées : 45,5\% pour les 6-12 ans et 35,9\% pour les 13-17 ans (tableau 2). La mise en parallèle des fréquences des groupes d'âge montre que les adolescents de 13 à 17 ans en situation de récurrence dont la sécurité ou le développement est actuellement compromis ont 1,4 fois plus de chance d'avoir déjà reçu des services du centre jeunesse par rapport aux adolescents qui ne sont pas en contexte de compromission. Lorsque l'on considère les situations d'abandon et de négligence, on note qu'elles regroupent plus d'un enfant sur deux $(52,3 \%)$ en situation de récurrence dont la sécurité ou le développement est compromis. Enfin, les résultats montrent que près des deux tiers $(64,3 \%)$ des enfants en récurrence avec une évaluation SDC avaient déjà reçu des services du centre jeunesse.

\section{CONCLUSION}

L'objectif de la présente étude est d'établir, selon les cinq cohortes regroupant tous les enfants dont le signalement est retenu pour évaluation, le taux de récurrence et de le ventiler en fonction du sexe de l'enfant, de son groupe d'âge et de la problématique évaluée. Les résultats montrent qu'un enfant sur quatre $(24,5 \%)$ est en situation de récurrence à la suite d'une évaluation de la DPJ confirmant le contexte de compromission; que les garçons $(55,6 \%)$ se retrouvent davantage dans cette situation que les filles (44,4\%); qu'un enfant sur deux en récurrence est un adolescent de 13 à 17 ans (50,2\%); et qu'il s'agit essentiellement d'une problématique d'abandon ou de négligence $(52,3 \%)$. Mais ce qui apparaît plus problématique encore, c'est le constat qu'un enfant sur six (15,8\%) en récurrence avait été antérieurement évalué et jugé comme vivant une situation de compromission, pour laquelle des services psychosociaux lui avaient été offerts durant une certaine période avant que le dossier ne soit fermé par la DPJ. II s'agit donc d'enfants et de familles qui s'étaient retrouvés en situation de compromission et qui doivent à nouveau y faire face malgré les interventions de la DPJ et du centre jeunesse. Un résultat similaire est obtenu par l'étude de Tourigny et al. (2002) sur l'incidence et les caractéristiques des situations signalées à la Direction de la protection de la jeunesse du Québec (Étude d'incidence québécoise, ÉIQ). Cette 
étude établit à 20,2\% la proportion des signalements jugés SDC qui ont fait l'objet d'une intervention des $\mathrm{CJ}$ au cours des cinq années précédentes. L'écart entre les deux proportions $(15,8 \%-20,2 \%)$ s'explique principalement par l'unité de mesure retenue par les études; l'ÉIQ prend comme base de calcul le nombre de signalements, alors que la nôtre s'appuie sur le nombre d'enfants signalés, éliminant ainsi les situations à signalement multiple.

Les résultats dégagés par la présente étude permettent d'obtenir pour une des premières fois un taux moyen de récurrence sur cinq années à la direction de la protection de la jeunesse d'une région administrative du Québec. Au regard de la stabilité des taux de récurrence observés à l'intérieur des cinq cohortes, on doit s'attendre à observer qu'environ $25 \%$ des enfants qui seront évalués et jugés en situation de compromission par la DPJ se retrouveront en situation de récurrence. Qui plus est, ce résultat confirme dans une certaine mesure les taux de récurrence observés par Sheriff et Berlinguet (1994), par Jacob et Laberge (2001) ou encore par Tourigny et al. (2002), à la différence que cette fois-ci il ne s'agit plus de taux basés sur des échantillons, mais bien sur des cohortes d'enfants à dossier fermé à la DPJ. Par ailleurs, les résultats de cette étude indiquent qu'au moment où la DPJ retient un signalement pour évaluation de la compromission, près d'un enfant sur cinq (19,6 \%) est déjà en situation de récurrence, c'est-à-dire que la DPJ avait par le passé effectué une évaluation de la compromission auprès de ces derniers. Un résultat similaire, soit un taux moyen de récurrence de 19,64\%, est obtenu par Fluke, Yuan et Edwards (1999) après avoir compilé tous les dossiers retenus en 1994 et 1995 par les agences de protection à l'enfance de dix États américains. Toutefois, ces auteurs mentionnent la présence d'une grande variabilité des taux entre les États, soit de $4,77 \%$ à $29,26 \%$. Un tel écart peut s'expliquer entre autres par des facteurs contextuels propres à chaque État américain.

Au regard des caractéristiques des enfants en situation de récurrence, certains résultats convergent, alors que d'autres se distinguent par rapport aux connaissances actuelles. L'étude laisse clairement entrevoir qu'un enfant sur deux $(50,2 \%)$ en situation de récurrence appartient au groupe d'âge des 12 à 17 ans. Cette proportion est similaire à celle obtenue par Sheriff et Berlinguet (1994), alors que des écarts marqués apparaissent dans les deux autres groupes d'âge : 15,6 \% (la présente étude) comparativement à $26 \%$ (Sheriff et Berlinguet) chez les 0 à 5 ans, et 34,2\% contre $23 \%$ chez les 6 à 12 ans. Par ailleurs, les proportions obtenues relativement aux groupes d'âge par notre étude sont à l'inverse de ceux obtenus par Fluke et al. (1999). En fait, du côté américain les plus hauts taux de récurrence s'observent chez les moins de 5 ans, alors que les taux les plus faibles sont notés chez les 12 à 17 ans. De telles disparités s'expliquent peut-être par des stratégies d'échantillonnage laissant place à l'introduction de biais ou à l'exclusion de certains motifs de référence (inclure ou pas, dans le calcul des taux de récurrence, les enfants qui manifestent des troubles de comportement) ou, encore, par des pratiques sociales différentes en protection de l'enfance, mais cela reste à vérifier empiriquement. Un autre écart important à noter est le pourcentage d'enfants maltraités en situation de récurrence. Nos résultats montrent que $60,7 \%$ de ces enfants (52,3\% pour abandon et négligence et $8,4 \%$ pour exploitation et abus) sont en récurrence, alors que des études américaine, anglaise ou 
canadienne situent le taux de récurrence chez les enfants maltraités entre $40 \%$ et 51 \% (DePanfilis et Zuravin, 1999; Hamilton et Browne, 1999; Trocmé et al., 2001). À notre avis, ces écarts ne sont que le reflet de définitions de la maltraitance des enfants qui varient selon les dispositions légales, les mandats juridiques, les pratiques professionnelles ainsi que les valeurs sociales et culturelles propres à chaque pays ou communauté (Trocmé et al., 2001). Finalement, aucune association n'a été observée entre le sexe de l'enfant en situation de récurrence et le groupe d'âge ou, encore, la situation retenue pour évaluation.

Bref, que l'on considère la récurrence au moment où les signalements sont retenus pour enquête ou encore à la fin du processus d'évaluation devant statuer à la présence ou à l'absence de compromission, c'est au moins un enfant sur six qui se retrouve en situation de récurrence. Comment expliquer un tel état de fait? Que devrait faire la DPJ, les centres jeunesse et leurs partenaires sociosanitaires pour parvenir à atténuer de telles situations vécues par les enfants et, par conséquent, faire baisser les taux de récurrence? Les réponses se trouvent-elles du côté des caractéristiques individuelles et familiales de cette clientèle, de la pratique professionnelle en protection de la jeunesse, de la qualité et de l'intensité de l'intervention par la DPJ ou encore dans l'organisation et la continuité des services sociaux et du soutien des organismes communautaires? II est possible qu'une conjoncture existe entre ces divers éléments pour rendre compte de la récurrence, mais cette hypothèse devra faire l'objet de nouvelles études.

\section{RÉFÉRENCES BIBLIOGRAPHIQUES}

BRODKIN, E.Z. (1997). «Inside the welfare contract: Discretion and accountability in State welfare administration », Social Service Review, vol. 71, $\mathrm{n}^{\circ}$ 1, p. 1-33.

DEPANFILIS, D., et S. ZURAVIN (1999). «Epidemiology of child maltreatment recurrences », Social Service Review, vol. 73, $\mathrm{n}^{\circ}$ 2, p. 218-239.

English, D.J., D.B. Marshall, S. Brummel et M. ORME (1999). «Characteristics of repeated referrals to Child Protective Services in Washington State », Child Maltreatment, vol. $4, \mathrm{n}^{\circ} 4$, p. 297-307.

FLUKE, J.D., Y.-Y.T. YUAN et M. EDWARDS (1999). « Recurrence of maltreatment: An application of the National Child Abuse and Neglect Data System (NCANDS) », Child Abuse and Neglect, vol. 23, $\mathrm{n}^{0} 7$, p. 633-650.

Fortin, L., D. LAFLEUR et A. ROBITAILLE (2002). Stratégie d'action pour les jeunes en difficulté et leur famille, Québec, ministère de la Santé et des Services sociaux, 47 p.

HAMILTON, C.C., et K.D. BRownE (1999). « Recurrent maltreatment during childhood: A survey of referrals to Police Child Protection Units in England », Child Maltreatment, vol. 4, $\mathrm{n}^{\circ} 4$, p. $275-286$.

INKELAS, M., et N. HALFON (1997). "Recidivism in child protective services », Children and Youth Services Review, vol. 19, n 3, p. 139-161. 
JACOB, M., et D. LABERGE (2001). « L'évaluation des signalements à la Direction de la protection de la jeunesse: étude des facteurs qui influencent les décisions prises par les intervenants », Criminologie, vol. 34, $n^{\circ} 1$, p. 123-154.

LESSARD, C. (2001). Indicateurs repères sur l'application de la Loi sur la protection de la jeunesse 1993-1994 à 1999-2000, Québec, ministère de la Santé et des Services sociaux, 243 p.

LESSARD, C. (2002). Indicateurs repères relatifs à l'application de la Loi sur la protection de la jeunesse 1993-1994 à 2000-2001, Québec, Service des indicateurs des mesures de la performance, ministère de la Santé et des Services sociaux.

MoRIN, H. (1993). Théorie de l'échantillonnage, Sainte-Foy, Les Presses de l'Université Laval, 178 p.

SHERIFF, T., et M. BERLINGUET (1994). Re-signalement et réévaluation des dossiers. La récurrence des bénéficiaires à la Direction de la protection de la jeunesse de Québec, Direction de la recherche et de l'enseignement, Centres jeunesse de Québec, $170 \mathrm{p}$.

SMITH, B.D., et S.E.F. Donovan (2003). «Child welfare practice in organizational and institutional context », Social Service Review, vol. 77, n 4, p. 541-563.

tourigny, M., M. Mayer, J. Wright, C. Lavergne, N. Trocmé, S. Hélie, C. Chamberland, C. Bouchard, R. Cloutier, M. Jacob, J. Boucher et M.-C. Larrivée (2002). Étude sur l'incidence et les caractéristiques des situations d'abus, de négligence, d'abandon et de troubles de comportement sérieux signalées à la Direction de la protection de la jeunesse du Québec (ÉIQ), Montréal, Centre de liaison sur l'intervention et la prévention psychosociales (CLIPP), $216 \mathrm{p}$.

TrocmÉ, N., B. FALLON, B. MACLAURIN et B. Copp (2002). Un système en transformation? Une analyse comparative des études ontariennes sur les signalements de mauvais traitements envers les enfants (OIS 1993/1998), Centre d'excellence pour la protection et le bien-être de l'enfant, Université de Toronto, $24 \mathrm{p}$.

Trocmé, N., B. Maclaurin, B. Fallon, J. Daciuk, D. Billingsley, M. Tourigny, M. Mayer, J. Wright, K. BARTER, G. BuRFORD, J. Hornick, R. Sullivan et B. McKenzie (2001). Étude canadienne sur l'incidence des signalements de cas de violence et de négligence envers les enfants : rapport final, Ottawa, ministère des Travaux publics et des Services gouvernementaux, $196 \mathrm{p}$. 